\title{
Coercivity enhancement of Dy-coated Nd-Fe-B flakes by crystallization
}

\author{
H. Fukunaga, ${ }^{1, a)}$ Y. Sugimoto, ${ }^{1}$ M. Nakano, ${ }^{1}$ T. Yanai, ${ }^{1}$ S. Ohta, ${ }^{2}$ M. Itakura, ${ }^{2}$ \\ and M. Nishida ${ }^{2}$ \\ ${ }^{1}$ Faculty of Engineering, Nagasaki University, Nagasaki 852-8521, Japan \\ ${ }^{2}$ Department of Applied Science for Electronics and Materials, Kyushu University, Kasuga, Fukuoka \\ 816-8580, Japan
}

(Presented 16 November 2010; received 30 September 2010; accepted 15 November 2010; published online 17 March 2011)

\begin{abstract}
The coercivity of isotropic Dy-coated Nd-Fe-B flakes was enhanced by crystallization and simultaneous diffusion of Dy from their surfaces. Amorphous Dy-coated Nd-Fe-B flakes were crystallized by heating them to $923 \mathrm{~K}$ 2over a 2 min period followed by rapid cooling. During crystallization, the Dy on the surface diffused into the flakes. This low-temperature rapid annealing produced flakes with fine grains and the Dy diffusion enhanced their coercivity. The coercivity after crystallization increased with increasing Dy layer thickness, although the remanence decreased when the layer thickness exceeded $3 \mu \mathrm{m}$. Thick coatings of over $6 \mu \mathrm{m}$ resulted in the formation of $\mathrm{DyFe}_{2}$, which degraded the magnetic properties of the crystallized flakes. Flakes with a 3- $\mu$ m-thick coating exhibited excellent magnetic properties after annealing: They had a coercivity of $1880 \mathrm{kA} / \mathrm{m}$ and a remanence of $78 \mathrm{emu} / \mathrm{g}$. This coercivity is approximately $500 \mathrm{kA} / \mathrm{m}$ higher than that of uncoated flakes, whereas the remanence is comparable to that of uncoated flakes. (C) 2011 American Institute of Physics. [doi:10.1063/1.3549604]
\end{abstract}

\section{INTRODUCTION}

Although Nd-Fe-B-based magnets have the highest energy product at room temperature of all of the magnets, their coercivity $H_{\mathrm{c}}$ drops drastically above $100{ }^{\circ} \mathrm{C}$ because of their low Curie temperature and low anisotropy field. ${ }^{1}$ This disadvantage can be overcome by partially substituting $\mathrm{Nd}$ in $\mathrm{Nd}_{2} \mathrm{Fe}_{14} \mathrm{~B}$ by a heavy rare-earth element such as Dy or $\mathrm{Tb}$ because both $\mathrm{Dy}_{2} \mathrm{Fe}_{14} \mathrm{~B}$ and $\mathrm{Tb}_{2} \mathrm{Fe}_{14} \mathrm{~B}$ have high anisotropy fields at high temperatures. ${ }^{2}$

However, substituting Nd with a heavy rare-earth element reduces the magnetization of magnets. Furthermore, because there are limited supplies of heavy rare-earth elements it is desirable to reduce their usage. One method of overcoming this problem is to diffuse a heavy rare-earth element only in the vicinity of grain surfaces. The coercivity of a sintered Nd-Fe-B-based magnet has been enhanced by diffusing a heavy rare-earth metal from its surface. ${ }^{3-6}$ Sintering has also been used to diffuse a heavy rare-earth element to $\mathrm{Nd}_{2} \mathrm{Fe}_{14} \mathrm{~B}$ grain surfaces. ${ }^{7,8}$ In both of these methods, the heavy rare-earth element is diffused by annealing at high temperatures of over $1000 \mathrm{~K}$. A low-temperature process is required for isotropic magnets that are fabricated by crystallizing an amorphous powder because hightemperature annealing causes $\mathrm{Nd}_{2} \mathrm{Fe}_{14} \mathrm{~B}$ grains to grow, which degrades the magnetic properties of isotropic magnet powders. ${ }^{9}$

In this study, we developed a low-temperature method for diffusing Dy into isotropic Nd-Fe-B crystalline flakes, thereby enhancing their coercivity.

\footnotetext{
a) Author to whom correspondence should be addressed. Electronic mail: fukunaga@nagasaki-u.ac.jp.
}

\section{EXPERIMENTAL PROCEDURES}

A $14-\mu$ m-thick Dy layer was deposited on one surface of amorphous $\mathrm{Nd}_{2.36} \mathrm{Fe}_{14} \mathrm{~B}_{1.05}$ flakes by pulsed laser deposition (PLD) in a vacuum. The Dy-coated flakes were then crystallized by heating them to $923 \mathrm{~K}$ followed by rapid cooling in an infrared furnace. Dy diffused into the flakes during crystallization. The heating rate was $462 \mathrm{~K} / \mathrm{min}$ and the Dy layer thickness was $1 \mu \mathrm{m}$ to12. Dy or Tb-coated amorphous $\mathrm{Nd}_{2.4} \mathrm{Fe}_{14} \mathrm{~B}$ films, approximately $16 \mu \mathrm{m}$ thick, were also prepared for observations with transmission electron microscopy (TEM) to analyze the microstructure produced by PLD. Dy and Tb were then diffused by applying the same annealing conditions as those used for the flakes.

The magnetic properties of the samples were measured using a vibrating sample magnetometer after magnetization under a pulsed field of $6.4 \mathrm{MA} / \mathrm{m}$. The sample microstructures were observed by TEM and their compositions were determined by energy-dispersive X-ray spectroscopy (EDS). The phases in the samples were analyzed by X-ray diffraction.

\section{RESULTS AND DISCUSSION}

Figure 1 shows hysteresis loops of uncoated $\mathrm{Nd}_{2.36} \mathrm{Fe}_{14} \mathrm{~B}_{1.05}$ flakes and $\mathrm{Nd}_{2.36} \mathrm{Fe}_{14} \mathrm{~B}_{1.05}$ flakes coated with 3- $\mu \mathrm{m}$-thick and $12-\mu \mathrm{m}$-thick Dy layers after crystallization. It clearly reveals that the Dy coating layer increases the coercivity $H_{\mathrm{c}}$. However, the $12-\mu \mathrm{m}$-thick coating reduced the remanence $M_{\mathrm{r}}$, and its demagnetization curve has a step in the low-field region, which suggests the presence of magnetically soft phases. The flakes coated with a $6-\mu$ m-thick Dy layer also had two-step demagnetization curves. Figure 2 shows plots of the obtained $H_{\mathrm{c}}$ and $M_{\mathrm{r}}$ as a function of the Dy layer thickness. The coercivity increased with increasing 


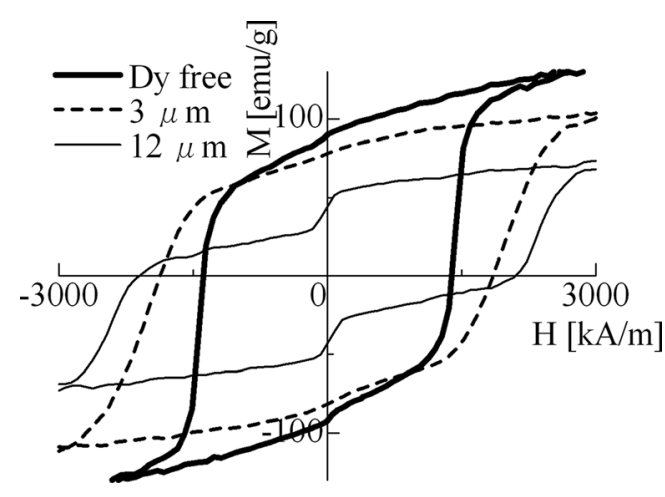

FIG. 1. $M-H$ loops for uncoated Nd-Fe-B flakes and Nd-Fe-B flakes coated with 3- and $12-\mu \mathrm{m}$-thick Dy layers after crystallization.

Dy layer thickness, and it reached $1880 \mathrm{kA} / \mathrm{m}$ at a thickness of $3 \mu \mathrm{m}$. A remarkable reduction in $M_{\mathrm{r}}$ was observed when the Dy layer thickness exceeded $3 \mu \mathrm{m}: M_{\mathrm{r}}$ of the flakes coated with a 3- $\mu \mathrm{m}$-thick Dy layer was nearly the same as that of the uncoated flakes.

Figure 3 shows X-ray diffraction patterns for uncoated $\mathrm{Nd}-\mathrm{Fe}-\mathrm{B}$ flakes and Nd-Fe-B flakes coated with a $12-\mu \mathrm{m}-$ thick Dy layer. The diffraction patterns of the Dy-coated flakes were obtained from the coated surfaces. The diffraction pattern of the as-quenched flakes indicates that they are amorphous. Peaks from Dy and $\mathrm{R}_{2} \mathrm{O}_{3}(\mathrm{R}=\mathrm{Dy}$ or $\mathrm{Nd})$ were observed after deposition of Dy, which suggests that a Dy layer was formed on the surfaces. A peak from $\mathrm{DyFe}_{2}$ appeared after crystallization. The thermomagnetic analysis results shown in Fig. 4 also confirm the presence of $\mathrm{DyFe}_{2}$. As $\mathrm{DyFe}_{2}$ has a cubic structure ${ }^{10}$ with a magnetic anisotropy constant of $2.1 \times 10^{6} \mathrm{~J} / \mathrm{m}^{3}$, this phase is considered to be responsible for the two-step demagnetization curve of this sample.

To confirm the diffusion of Dy, cross sections of flakes were observed by scanning electron microscopy (SEM) and the depth profile of the Dy content was analyzed by EDS. Figure 5 shows the Dy, Nd, and Fe contents (the B content was omitted from this analysis) in a crystallized flake as a function of depth from the surface. The Dy layer is approximately $2.5 \mu \mathrm{m}$ thick, as indicated by the profile. Dy diffused

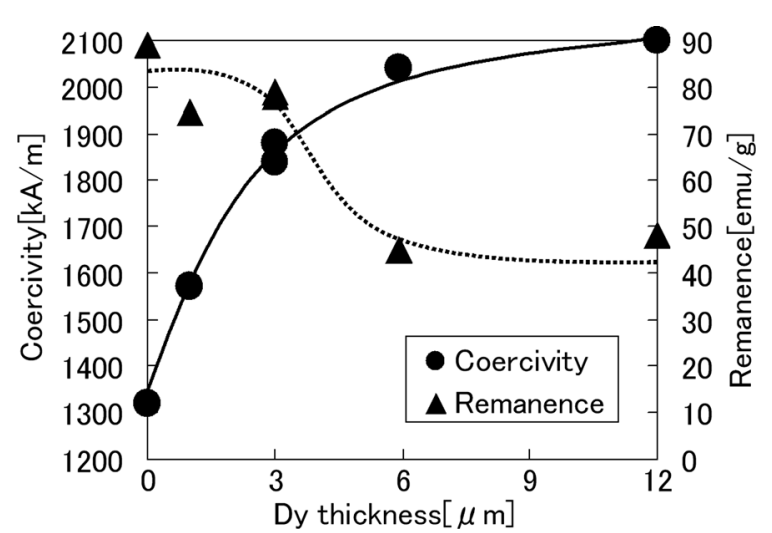

FIG. 2. Coercivity and remanence of Dy-coated flakes as a function of Dy layer thickness.

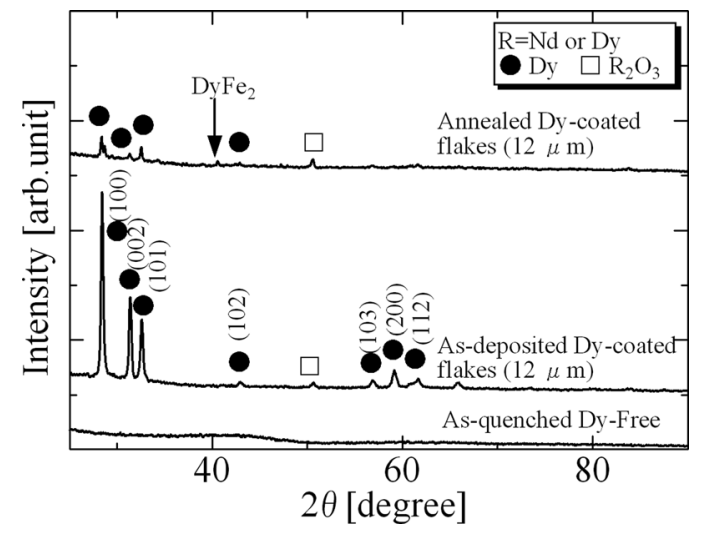

FIG. 3. X-ray diffraction patterns of uncoated Nd-Fe-B flakes and Nd-Fe-B flakes coated with a $12-\mu \mathrm{m}$-thick Dy layer. The patterns of the coated flakes were obtained from the Dy-coated surfaces.

up to $6.5 \mu \mathrm{m}$ into the flake despite a very short annealing time.

Further analysis was performed for $16-\mu \mathrm{m}$-thick $\mathrm{Nd}_{2.4} \mathrm{Fe}_{14} \mathrm{~B}$ films coated with a Dy or Tb layer. After crystallization at $923 \mathrm{~K}$, both the Dy and $\mathrm{Tb}$ layers increased $H_{\mathrm{c}} ; H_{\mathrm{c}}$ of the $\mathrm{Nd}_{2.4} \mathrm{Fe}_{14} \mathrm{~B}$ film with a $2-\mu \mathrm{m}$ thick Tb layer exceeded $2000 \mathrm{kA} / \mathrm{m}$. Figure 6 shows typical TEM images of this film. The sample was composed of fine grains and the map of the Tb-M line indicates that $\mathrm{Tb}$ is concentrated in the vicinity of grain boundaries, which agrees with previous observations of sintered magnets. $^{3-6}$ Detailed results for the TEM observations have been published elsewhere. ${ }^{11}$ As $\mathrm{Tb}$ and Dy have similar diffusion behaviors, ${ }^{5}$ the Dy in the present Dy-coated flakes is expected to be concentrated in the vicinity of grain boundaries after crystallization. Therefore, the observed $H_{\mathrm{c}}$ enhancement of these flakes is attributed to the diffusion of Dy through the grain boundaries.

\section{CONCLUSIONS}

Amorphous Nd-Fe-B flakes coated with a Dy layer were crystallized. Their coercivity $H_{\mathrm{c}}$ was enhanced by diffusion of Dy from their surfaces during crystallization. SEM and

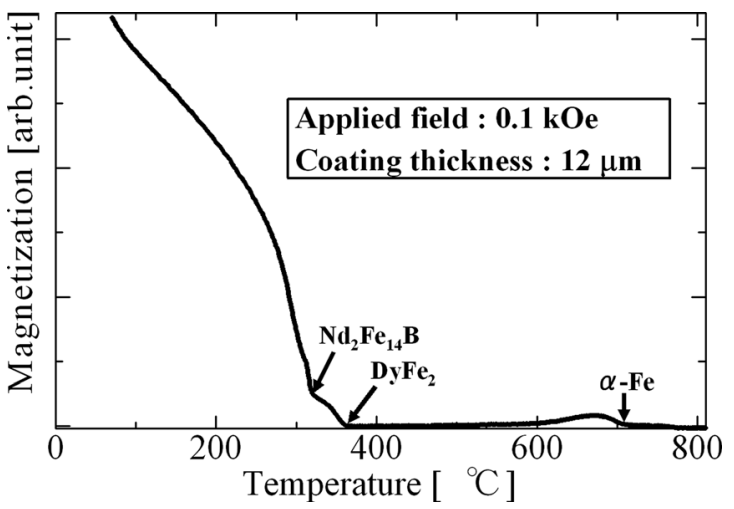

FIG. 4. Thermomagnetic properties of Dy-coated flakes after crystallization. The arrows indicate the Curie temperatures of $\mathrm{Nd}_{2} \mathrm{Fe}_{14} \mathrm{~B}$, $\mathrm{DyFe}_{2}$, and $\alpha-\mathrm{Fe}$. 


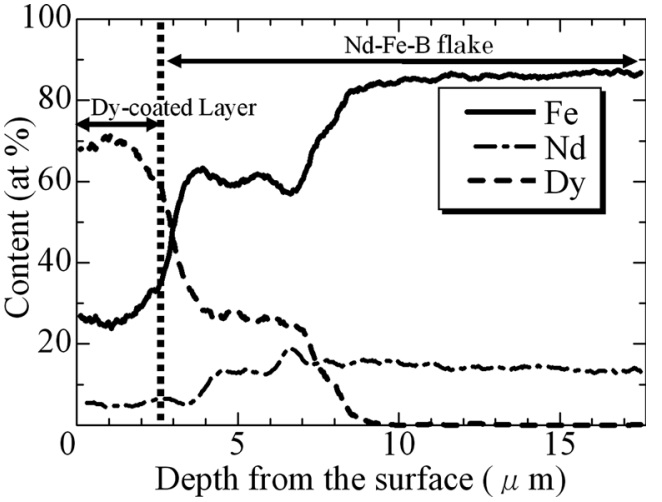

FIG. 5. Depth profile of Dy, Nd, and Fe contents in a flake after crystallization. The Dy layer is approximately $2.5 \mu \mathrm{m}$ thick.

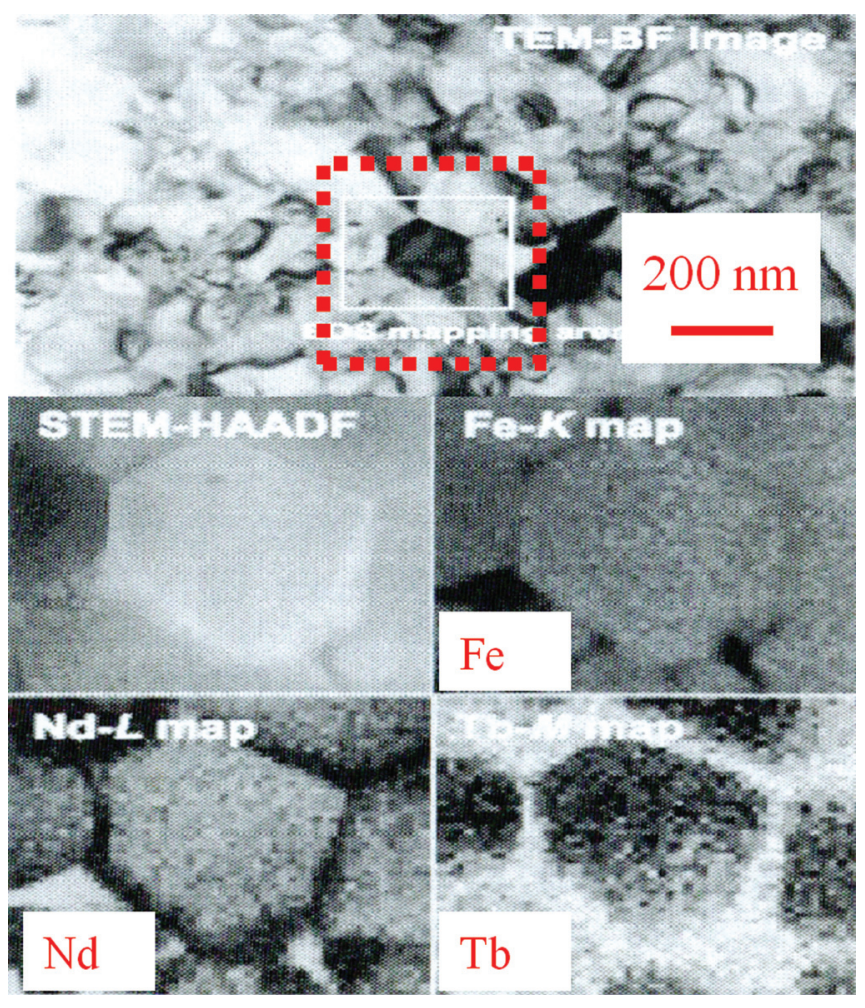

FIG. 6. (Color online) Typical TEM images of Tb-coated $\mathrm{Nd}_{2.4} \mathrm{Fe}_{14} \mathrm{~B}$ film after crystallization. The lower part shows images obtained using $\mathrm{Fe}-\mathrm{K}$, Nd-L, and Tb-M lines. Detailed results of TEM observations have been published elsewhere (Ref. 11).
EDS analyses confirmed that Dy diffuses into the flakes. This investigation used a much lower annealing temperature $(932 \mathrm{~K})$ and a much shorter annealing time $(0 \mathrm{~min})$ than those employed for diffusing Dy in sintered magnets. This enabled us to obtain isotropic Nd-Fe-B-based flakes consisting of fine grains with a high $H_{\mathrm{c}}$.

The coercivity after crystallization increased with increasing Dy layer thickness, although $M_{\mathrm{r}}$ decreased when the coating thickness exceeded $3 \mu \mathrm{m}$. Coatings that were more than $6 \mu \mathrm{m}$ thick resulted in the formation of $\mathrm{DyFe}_{2}$, which deteriorated the magnetic properties of the crystallized flakes. Flakes coated with a $3-\mu$ m-thick layer exhibited excellent magnetic properties $\left(H_{\mathrm{c}}=1880 \mathrm{kA} / \mathrm{m}\right.$ and $M_{\mathrm{r}}=78$ emu/g). This $H_{\mathrm{c}}$ value is approximately $500 \mathrm{kA} / \mathrm{m}$ higher than that of the uncoated flakes, whereas $M_{\mathrm{r}}$ is comparable to that of the uncoated flakes.

\section{ACKNOWLEDGMENTS}

We thank Dr. T. Iriyama and Dr. S. Suzuki of Daido Steel Co., Ltd. for kindly supplying the Nd-Fe-B flakes. This work was supported in part by the Ministry of Education Science Sports and Culture of Japan MESSC(JP) under a Grant-in-Aid (22360128).

${ }^{1}$ E. B. Boltich, E. Oswald, M. Q. Huang, D. Hirosawa, W. E. Wallace, and E. Burzo, J. Appl. Phys. 57, 4106 (1985).

${ }^{2}$ M. Sagawa, S. Hirosawa, H. Yamamoto, S. Fujimura, and Y. Matsuura, Jpn. J. Appl. Phys. 26, 785 (1987).

${ }^{3}$ H. Nakamura, K. Hirota, T. Minowa, and M. Honshima, IEEE Trans. Magn. 42, 2909 (2006).

${ }^{4}$ H. Nakamura, K. Hirota, T. Minowa, and M. Honshima, J. Magn. Soc. Jpn. 31, 6 (2007).

${ }^{5}$ D. S. Li, S. Suzuki, T. Kawasaki, and K. Machida, Jpn. J. Appl. Phys. 47, 7876 (2008).

${ }^{6}$ H. Suzuki, Y. Satsu, and N. Komoto, J. Appl. Phys. 105, 07A734 (2009).

${ }^{7}$ Q. Liu, L. Zhang, X. Dong, D. Xu, and M. Komuro, Scr. Mater. 61, 1048 (2009).

${ }^{8}$ T. Hidaka, C. Ishizaka, and M. Hosaka, Proceedings of the 21st Workshop on RE Permanent Magnets and Their Applications, pp. 100102 (2010).

${ }^{9}$ H. Fukunaga, K. Ihara, and K. Narita, J. Magn. Soc. Jpn. 10, 229 (1986).

${ }^{10}$ A. Clark, J. Cullen, and K. Sato, AIP Conf. Proc. No. 24, 670 (1975).

${ }^{11}$ M. Ishimaru, M. Itakura, M. Nishida, M. Nakano, and H. Fukunaga, Mater. Trans. 51, 1939 (2010). 